\title{
COMMENTARY
}

\section{An interesting application of lung ultrasonography}

\author{
Paul H Mayo* \\ See related research by Raimondi et al., http://ccforum.com/content/16/6/R220
}

\begin{abstract}
In the previous issue of Critical Care, Raimondi and colleagues investigate whether lung ultrasonography has utility for the assessment of respiratory distress in the neonate. This commentary reviews the results and implications of their study.
\end{abstract}

Lung ultrasonography has strong utility in adult critical care medicine, where it is a key element in the diagnosis and management of respiratory failure. In their welldesigned study, Raimondi and colleagues [1] describe an interesting application of lung ultrasonography to neonatology. They used ultrasonography to examine the lungs of babies at birth and serially at 24 and 36 hours post-delivery. They found a close correlation between the results of lung ultrasonography and the development of respiratory distress (RD) of the newborn. The positive and negative predictive value of the test indicates that it has major value in identifying neonates who are at risk for RD following delivery. Their results raise the consideration that ultrasonography should become the primary imaging modality for assessment of the neonatal lung. Given this possibility, Lichtenstein $[2,3]$ has determined that the findings of lung ultrasonography in the neonatal population are identical to those found in the adult patient. Such key findings as A lines, B lines, consolidation, pleural effusion, or lung sliding, which have been described in the seminal work of Dr Lichtenstein [4], apply equally to the adult as to the neonate. When it comes to lung ultrasonography, a baby is simply an adult in miniature. The critical care clinician may confidently use the principles of lung ultrasonography across the entire age spectrum.

When scanning the adult patient, the intensivist may face impediments in performing a comprehensive thoracic examination. The patient is in the supine position and may be difficult to position for a full examination, making

\footnotetext{
*Correspondence: mayosono@gmail.com
}

Long Island Jewish Medical Center, New Hyde Park, NY 11040, USA it difficult to examine the postero-lateral thorax. Obesity, edema, or heavy musculature may degrade image quality. These are not considerations with the neonate, where the examination is characterized by ease of use. As the depth of examination is only a few centimeters, a high frequency transducer is used to yield images of excellent resolution. The transducer may be swept over the thorax in a series of scan lines to yield a high resolution image set of the entire lung. The examination may be easily repeated as often as needed. A portable ultrasound machine may be used to scan the lungs of multiple patients in the neonatal intensive unit and the delivery area for immediate assessment of risk for RD. The machine is not limited to use for scanning the lung and pleura, as ultrasonography has many other applications for the neonatologist and the pediatrician.

A special reason to consider that ultrasonography should be the primary imaging modality for the neonate (or for all pediatric patients), is that it reduces the exposure of patients to ionizing radiation. The pediatric patient is at particular risk for the carcinogenic effects of radiation exposure. Every time that an ultrasound examination replaces a standard radiograph or a CT scan, benefit accrues to the patient in terms of reducing risk of cancer later in their life. The importance of this issue cannot be overstated.

One of the practical impediments to the widespread use of ultrasonography is that some intensivists may still consider it as a traditional radiographic procedure, that is, an examination that is ordered by the bedside clinician but that is delayed in its performance, delayed in its interpretation, and interpreted by an offline reader with no deep understanding of the case. Intensivists should deploy ultrasonography using a different paradigm. The scan is done at point of care by the frontline clinician. The images are immediately interpreted by the clinician, and the results are immediately applied within the context of full knowledge of the clinical situation. The indicated ultrasound is repeated as often as is clinically indicated in order to observe the trajectory of the disease process, to guide management, and to examine for additional diagnosis or complications. While the study of Raimondi and colleagues shows that lung ultrasonography has excellent utility for assessment of RD in the neonate, 
it also presents to the critical care community an excellent example of point of care ultrasonography performed by the clinician and used for immediate clinical judgment call. It is an excellent example of the paradigm that brings ultrasonography to its best place: at the bedside of the patient.

The study shows that lung ultrasonography may replace standard radiography for assessment of neonatal RD; and, by implication, for assessment of patients with other forms of respiratory failure in the critical care unit. Several European groups have done impressive work in defining the utility of lung ultrasonography in critical care medicine [5-13]. It is possible that critical care ultrasonographers may declare the death of the standard chest radiograph $[14,15]$. The usual intensive care unit chest radiograph, supine, rotated, and with uncertain penetration; is far outclassed by lung ultrasonography. Similarly, lung ultrasonography may largely replace chest CT with its heavy radiation exposure. Only then will ultrasonography reach its full potential at the bedside of the critically ill.

To reach that point, faculty must recognize that a major challenge with lung ultrasonography remains that it requires training. Raimondi and colleagues are expert level ultrasonographers. All intensivists should be at their level. The issue is to design training programs that assure competence for intensivists in this key aspect of critical care ultrasonography

Abbreviations

$R D$, respiratory distress.

\section{Competing interests}

The author declares that he has no competing interests.

Published: 30 January 2013

\section{References}

1. Raimondi F, Migliaro F, Sodano A, Umbaldo A, Romano A, Vallone G, Capasso $\mathrm{L}$ : Can neonatal lung ultrasound monitor fluid clearance and predict the need of respiratory support? Crit Care 2012, 16:R220.
2. Lichtenstein DA: Ultrasound examination of the lungs in the intensive care unit. Pediatr Crit Care Med 2009, 10:693-698.

3. Lichtenstein DA: Applications of Lung Ultrasound in the Critically III: Lung Ultrasound in the Neonate. In General Ultrasound in the Critically III. Berlin, Germany: Springer-Verlag; 2010:203-208.

4. Lichtenstein DA: Section on Lung Ultrasonography Chapters 14-20. In General Ultrasound in the Critically III. Berlin, Germany: Springer-Verlag; 2010:117-201.

5. Lichtenstein D, Goldstein I, Mourgeon E, Cluzel P, Grenier P, Rouby JJ: Comparative diagnostic performances of auscultation, chest radiography, and lung ultrasonography in acute respiratory distress syndrome. Anesthesiology 2004, 100:9-15.

6. Lichtenstein DA, Mezière GA: Relevance of lung ultrasound in the diagnosis of acute respiratory failure: The BLUE Protocol. Chest 2008, 134:117-125.

7. Mathis G, BlankW, Reissig A, Lechleitner P, Reuss J, Schuler A, Beckh S: Thoracic ultrasound for diagnosing pulmonary embolism: a prospective multicenterstudy of 352 patients. Chest 2005, 128:1531-1538.

8. Arbelot C, Ferrari F, Bouhemad B, Rouby JJ: Lung ultrasound in acute respiratory distress syndrome and acute lung injury. Curr Opin Crit Care 2008, 14:70-74.

9. Copetti R, Soldati G, Copetti P: Chest sonography: a useful tool to differentiate acute cardiogenic pulmonary edema from acute respiratory distress syndrome. Cardiovascular Ultrasound 2008, 6:16-26.

10. Volpicelli G, Caramello V, Cardinale L, Mussa A, Bar F, Mauro F: Bedside ultrasound of the lung for the monitoring of acute decompensated heart failure. Am J Emerg Med 2008, 26:585-591.

11. Bouhemad B, Liu ZH, Arbelot C, Zhang M, Ferarri F, Le-Guen M, Girard M, Lu Q, Rouby JJ: Ultrasound assessment of antibiotic-induced pulmonary reaeration in ventilator-associated pneumonia. Crit Care Med 2010, 38:84-92.

12. Bouhemad B, Brisson H, Le-Guen M, Arbelot C, Lu Q, Rouby JJ: Bedside ultrasound assessment of positive end-expiratory pressure-induced lung recruitment. Am J Respir Crit Care Med 2011, 183:341-347.

13. Caiulo VA, Gargani L, Caiulo S, Fisicaro A, Moramarco F, Latini G, Picano E, Mele G: Lung ultrasound characteristics of community-acquired pneumonia in hospitalized children. Pediatr Pulmonol 2012. doi: 10.1002/ppul.22585.

14. Ioos V, Galbois A, Chalumeau-Lemoine L, Guidet B, Maury E, Hejblum G: An integrated approach for prescribing fewer chest x-rays in the ICU. Ann Intensive Care 2011. doi: 10.1186/2110-5820-1-4.

15. Zanobetti $M$, Poggioni $C$, Pini R: Can chest ultrasonography replace standard chest radiography for evaluation of acute dyspnea in the ED? Chest 2011, 139:1140-1147.

doi:10.1186/cc11914

Cite this article as: Mayo PH: An interesting application of lung

ultrasonography. Critical Care 2013, 17:114. 